\title{
Updated GOLD COPD Recommendations
}

\author{
An expert interview with Mario Cazzola
}

Professor of Respiratory Medicine, University of Rome Tor Vergata, Italy

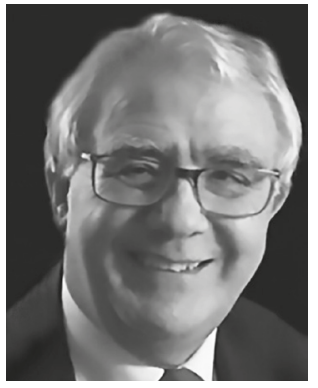

\section{Mario Cazzola}

Mario Cazzola is an Honorary Professor of Respiratory Medicine at the University of Rome Tor Vergata, Italy, and Visiting Professor at the Sackler Institute of Pulmonary Pharmacology, GKT School of Biomedical Sciences, London, UK. He is a fellow of the European Respiratory Society and has received the 2015 European Respiratory Society Lifetime Achievement Assembly Award.

DOl: https://doi.org/10.17925/ERPD.2017.03.01.17

\section{Keywords}

Chronic obstructive pulmonary disease, Global Initiative for Chronic Obstructive Lung Disease recommendations, definition, assessment, treatment, escalating or de-escalating therapy

Disclosure: Mario Cazzola has participated as a speaker, and advisor in scientific meetings and courses under the sponsorship of Almirall, AstraZeneca, Biofutura, Boehringer Ingelheim, Chiesi Farmaceutici, GlaxoSmithKline, Menarini Group, Lallemand Mundipharma, Novartis, Pfizer, Verona Pharma, and Zambon, and is or has been a consultant to Chiesi Farmaceutici, Lallemand, Novartis, Verona Pharma, and Zambon. This is an expert interview piece and as such has not undergone the journal's standard peer review process. No funding was received for the publication of this article.

Authorship: All named authors meet the Internationa Committee of Medical Journal Editors (ICMJE) criteria for authorship of this manuscript, take responsibility for the integrity of the work as a whole, and have given final approval for the version to be published. open Access: This article is published under the Creative Commons Attribution Noncommercial License which permits any non-commercial use, distribution, adaptation and reproduction provided the original author(s) and source are given appropriate credit.

Received: 25 January 2017

Published Online: 26 July 2017

Citation: European Respiratory \& Pulmonary Diseases, 2017:3(1):17-8

Corresponding Author: Mario Cazzola, Dipartimento di Medicina dei Sistemi, Università di Roma Tor Vergata, Via Montpellier 1, Rome 00133, Italy.

E:mario.cazzola@uniroma2.it

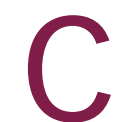
hronic obstructive pulmonary disease (COPD) is currently the fourth leading cause of death worldwide. In order to minimize the impact of COPD on public health, there is a need for preventative strategies and appropriate methods of management. The Global Initiative for Chronic Obstructive Lung Disease (GOLD) has been developing international guidelines for COPD since 1997. Recently, updated 2017 GOLD guidelines have been released; these include a revised definition of COPD. In an expert interview, Professor Mario Cazzola of the University of Rome Tor Vergata, Italy, discusses the key changes in the new recommendations.

\section{Q: The new GOLD recommendations have revised the definition of chronic obstructive pulmonary disease. Could you describe the revised definition and the rationale for the change?}

I must premise that the GOLD strategy includes recommendations and should not be interpreted as a guideline. Surely we must feel indebted to those who have, over time, developed the GOLD Strategy, but we must also be aware that there are points that are not the unanimous opinion of all experts and clinicians involved in the management of patients with chronic obstructive pulmonary disease (COPD).

The latest definition of COPD is 'a common, preventable and treatable disease that is characterised by persistent respiratory symptoms and airflow limitation that is due to airway and/or alveolar abnormalities usually caused by significant exposure to noxious particles or gases. The chronic airflow limitation that characterises COPD is caused by a mixture of small airways disease (for example, obstructive bronchiolitis) and parenchymal destruction (emphysema), the relative contributions of which vary from person to person. Chronic inflammation causes structural changes, small airways narrowing and destruction of lung parenchyma. A loss of small airways may contribute to airflow limitation and mucociliary dysfunction, a characteristic feature of the disease. Chronic respiratory symptoms may precede the development of airflow limitation and be associated with acute respiratory events. Chronic respiratory symptoms may exist in individuals with normal spirometry and a significant number of smokers without airflow limitation have structural evidence of lung disease manifested by the presence of emphysema, airway wall thickening and gas trapping."1

If I am correctly interpreting the new definition, I understand that now it is stated that COPD is a disease that can be treated and not that it is potentially treatable. Certainly, the therapeutic approach to COPD has greatly improved in recent years, but I believe that we are still far from being able to say that COPD is a treatable disease. 
The primary importance that is now given to persistent respiratory symptoms represents a fundamental change. Apparently, we have moved over time from considering lung function as the central part of the diagnosis and key element that helps in directing therapy, to assigning it a seemingly secondary role. I have always thought that the role previously assigned to the lung function was excessive because a patient never asks to be visited because of a low FEV ${ }_{1}$, but because he or she is suffering from breathlessness, limitation in performing an exercise and also because of frequent exacerbations. Nevertheless, in my view, regular monitoring of lung function is required to follow the evolution of the lung damage over time, but also to identify the COPD phenotype we are dealing with.

It is surprising that there is only very little mention of chronic inflammation. Nowadays, we know that the characteristics of inflammation are not always the same in all patients and that a definition of the specific endotype is essential for a proper treatment approach, but this does not mean we that should not consider inflammation as a key element in the natural history of COPD. Actually, oxidative stress, which is an important amplifying mechanism, triggers a well known inflammatory process. I am also very surprised that exacerbations and comorbidities are no longer mentioned in the definition of the disease. Nevertheless, the choice of treatment is certainly conditioned by the fact that the patient to be treated is or is not a frequent exacerbator and, in any case, we must always adjust therapy taking into account the comorbidities that in a patient with COPD are very frequent.

However, it seems fair to me to point out that in the GOLD document, all of these aspects are discussed, but many physicians often focus only on definition and this can be misleading.

\section{Q: What changes have been made to the airway, breathing, circulation, disability, exposure assessment tool?}

Although spirometry in conjunction with patient symptoms and exacerbation history, remains vital for the diagnosis, prognostication and consideration of other important therapeutic approaches, a refinement of the airway, breathing, circulation, disability, exposure (ABCDE) assessment tool has been suggested, which separates spirometric grades from the ABCDE groups. For some therapeutic recommendations, $A B C D E$ groups will be derived exclusively from patient symptoms and their history of exacerbation. This is not a real surprise because we cannot forget that in the UNLOCK study, 12\% of GOLD I (mild COPD) European patients in primary care were frequent exacerbators (two or more acute exacerbations of COPD [AECOPD] yearly), and $34 \%$ exacerbated at least once yearly. ${ }^{2}$ Furthermore, evidence proves that patients can shift from one category (frequent exacerbator or infrequent exacerbator) to another, but there is no clear explanation or marker for this shift, apart from the fact that patients on the borderline between infrequent and frequent AECOPDs are more likely to change category and this trend is regardless of the value of spirometry.

I am still very surprised that both the modified Medical Research Council (mMRC) scale and the COPD assessment test (CAT) are considered to assess symptoms. As is well explained in the document, these tools measure different things, therefore it would be necessary to clarify when to use one rather than the other and not leave the choice to the arbitrary decision of physicians.

\section{Q: What other key changes have been made in the new recommendations?}

I believe that two fundamental changes introduced in the therapeutic recommendations should be highlighted. First of all, the role of the dual bronchodilation (long-acting beta agonists [LABA]/long-acting muscarinic receptor antagonists [LAMA] fixed-dose combinations) is emphasised. The proof that dual bronchodilation can prevent or at least delay the onset of COPD exacerbations is extremely important, but raises the fundamental question of whether and/or when it is appropriate to switch patients with COPD from a LABA/ inhaled corticosteroid (ICS) regimen, which is still widely used to prevent exacerbations, to a LABA/LAMA one on the basis of the improvement in lung function and the lower exacerbation rates, or there is a subgroup of patients with COPD who may benefit the most from dual bronchodilation. Furthermore, we still do not know whether dual bronchodilation is effective in preventing COPD exacerbations regardless of their nature, although I must highlight that the baseline blood eosinophil count (blood eosinophil count $\geq 2 \%$ is a promising biomarker of response to ICSs in patients with COPD) still does not appear to predict what would be the most effective treatment regimen. It is essential to establish whether LABA/LAMA combination therapy is preferred over triple therapy (LAMA/LABA/ICS), and whether addition of an ICS to the LABA/LAMA combination provides additional clinical value.

This last is a fundamental question, since the last GOLD report suggests the possibility of escalating or de-escalating treatment according to the patient's clinical course. I completely agree that, in general, there is a large and often inappropriate use of ICS/LABA combination and, in any case, the withdrawal of the ICS in COPD patients at low risk of exacerbation can be safe, provided that patients are under regular treatment with long-acting bronchodilators. Maximising the treatment in patients with a degree of clinical instability by including an ICS in the therapeutic regimen is useful to control the disease, but may not be needed during periods of clinical stability.

In my opinion, for patients with severe but stable COPD, the withdrawal of ICS from triple therapy is possible, but not when the patient has been hospitalised for an acute exacerbation of COPD. It must still be established how long one should wait before withdrawing the ICS. Moreover, it is still unclear whether the same is true when only the LABA or the LAMA is withdrawn while continuing treatment with the other bronchodilator and the ICS. In any case, I strongly believe that it is always better to avoid a therapeutic step-up progression when it is not needed rather than being forced subsequently into a step-down approach in which the outcome is always unpredictable. All these considerations are absent in the 2017 GOLD report and this lack makes it impossible to establish with good approximation the value of escalation and de-escalation of therapy in COPD..

1. Vogelmeier CF, Criner GJ, Martinez FJ, et al., Global Strategy for the Diagnosis, Management, and Prevention of Chronic Obstructive Lung Disease 2017 Report: GOLD Executive Summary, Eur Respir J, 2017;49. pii: 1700214. doi: 10.1183/13993003.00214-2017.

2. Kruis AL, Ställberg B, Jones RC, et al., Primary care COPD patients compared with large pharmaceutically-sponsored COPD studies: an UNLOCK validation study, PLOS One, 2014;9:e90145. 